\title{
Ethnologies
}

\section{Jeux sportifs, codes et construction identitaire}

Règles explicites des mécanismes internes et règles implicites du pratiquant

\section{Marie Level, Éric Dugas et Thierry Lesage}

Volume 32, numéro 1, 2010

Jouer

Play

URI : https://id.erudit.org/iderudit/045215ar

DOI : https://doi.org/10.7202/045215ar

Aller au sommaire du numéro

Éditeur(s)

Association Canadienne d'Ethnologie et de Folklore

ISSN

1481-5974 (imprimé)

1708-0401 (numérique)

Découvrir la revue

Citer cet article

Level, M., Dugas, É. \& Lesage, T. (2010). Jeux sportifs, codes et construction identitaire : règles explicites des mécanismes internes et règles implicites du pratiquant. Ethnologies, 32(1), 113-132. https://doi.org/10.7202/045215ar

\section{Résumé de l'article}

L'inscription dans une activité sportive est vectrice d'identité au même titre que l'engagement politique ou la pratique d'une religion. Le pratiquant, placé dans un contexte culturel donné, ici les activités ludomotrices, est amené à incorporer un système de codes, de valeurs et de représentations véhiculés par des logiques internes d'une part et des mécanismes implicites d'autre part. C'est au travers du filtre d'analyse, qui conjugue l'étude du pratiquant et des structures dans lesquelles il agit, que nous explorons ici l'identité jouée dans les jeux et les sports afin de mieux appréhender les conditions d'expression et d'émancipation du joueur agissant. 


\title{
JEUX SPORTIFS, CODES ET CONSTRUCTION IDENTITAIRE
} Règles explicites des mécanismes internes et règles implicites du pratiquant

\author{
Marie Level \\ Éric Dugas \\ Thierry Lesage \\ Université Paris Descartes \\ GEPECS (EA 3625)
}

Parmi les multiples facettes qui composent l'identité, pratiquer une activité ludomotrice ${ }^{1}$ apparaît comme une composante significative. Toutefois, s'interroger sur le rapport entre les codes qui régissent les pratiques ludomotrices et la construction identitaire du pratiquant suppose au préalable une prise en compte du caractère protéiforme de la notion d'identité. Cette dernière renvoie en effet aussi bien au concept de personnalité qu'aux phénomènes de construction sociale à l'échelle de l'individu, du groupe et de la société. Loin d'une définition qui tendrait vers une réification de l'identité en un attribut immuable de la personne, il s'agira ici d'envisager avant tout le processus dynamique par lequel le pratiquant ${ }^{2}$ se construit et se reconnaît comme tel, dans

1. Les activités ludomotrices correspondent à l'ensemble des jeux (y compris les sports) dont la pertinence est avant tout motrice.

2. Nous établissons une distinction conceptuelle entre « joueur » et «sportif ». En effet, nous limitons le sport à un type particulier de jeu dont la spécificité réside dans quatre composantes définitoires sine qua none : la pertinence motrice, la règle, l'institutionnalisation, la compétition. De fait, si tous les sportifs sont des joueurs, l'inverse ne vaut pas. Aussi assidu et investi que soit le joggeur, sa pratique n'entre pas dans cette définition du sport même si elle en présente des «traits analogues » (Callède 2007). Enfin, le joggeur comme l'athlète seront ici qualifiés de «pratiquant». Il ne s'agit pas d'opérer une dichotomie entre le sérieux et le ludique qui opposerait la rigueur au plaisir puisque ces deux composantes peuvent être présentes de part et d'autre mais bien de construire un concept qui prémunit d'une définition «fourre-tout» inopérante. 
son rapport au monde et aux autres, au fil de ses expériences et interactions. Dans cette perspective, l'attachement à un jeu sportif sera envisagé comme levier de subjectivation : le sujet agissant se construit et s'exprime au travers du champ culturel spécifique au sein duquel il évolue et manifeste son intentionnalité. Ses actions sont alors à appréhender à la lumière d'un système de contraintes où s'articulent deux types de codes. D'une part, il existe des codes qui sont de nature explicite, c'est-à-dire les règles sur lesquelles s'appuie une logique interne à l'activité. D'autre part, certains relèvent de l'implicite, à l'image des normes et valeurs véhiculées plus tacitement, telles que les normes corporelles, le goût de l'effort, du dépassement voire de la douleur mais aussi les conduites informelles réinterprétée subjectivement (dans l'action de jeu, sur les plans techniques et tactiques, et à côté, comme dans des attitudes ritualisées). Ces dernières nous amènent notamment à réfléchir aux mécanismes par lesquels un sujet s'imprègne de manières d'être, d'agir et de penser lorsqu'il est placé dans le contexte de la pratique qu'il exerce ${ }^{3}$. Dit autrement, notre propos - inspirée de l'approche foucaldienne - est ici de questionner la constitution du sujet agissant dans un cadre plus ou moins normé.

Quelles que soient les valeurs attribuées au sport - catharsis, « opium du peuple » (Bröhm 2006), pacification de l'individu (Elias et Dunning 1994), etc. - les débats se focalisent essentiellement sur les pratiques physiques compétitives et institutionnalisées, laissant dans l'ombre les autres formes de pratiques. Or, aux deux pôles du continuum de l'espace ludomoteur, se placent d'un côté les activités inorganisées et libres, et de l'autre les activités formelles, compétitives et institutionnalisées à l'échelle mondiale (le sport). En effet, certaines activités telles que les pratiques urbaines (le streetbasket, les jeux sur city-stade, les pratiques en skateparks...) ont la particularité d'être autocodifiées, c'est-à-dire que les participants peuvent fixer à l'amiable des règles ponctuelles, fluctuantes et dans un espace et une temporalité qu'ils choisissent. A contrario, les disciplines circonscrites à la classe des sports sont soumises à des règles impérieuses dont l'entière autorité est du ressort des fédérations internationales. Entre ces deux modèles, se déploie une kyrielle de pratiques diversifiée : certaines se limitent à une institutionnalisation très localisée (lutte sénégalaise, boule lyonnaise...)

3. Ces mécanismes d'imprégnation sont à l'œuvre dans le cadre de la pratique sportive, mais ils ne s'y limitent pas. Ils sont constitutifs de l'identité globale et unifiée du sujet qui ne se défait pas pour autant de tout un pan de sa personne lorsqu'il agit dans un autre contexte que celui traité ici. 
tandis que d'autres, bien qu'extrêmement codifiées, ne sont pas sous le joug d'une instance officielle (ballon prisonnier, thèque...). Si les pratiques du patrimoine régional mettent l'accent sur la singularité ethnologique, l'uniformisation de la référence culturelle induite par la mondialisation du sport n'annihile pas pour autant tout enjeu identitaire. Loin d'empêcher l'émergence d'hétérogénéité, celle-ci se distille au contraire à tous les niveaux. En premier lieu, le sport, produit culturel et miroir de nos sociétés modernes, n'est pas homogène en son sein : il existe bien des cultures sportives propres à chaque discipline, c'est-àdire des systèmes de manières de penser, de sentir et d'agir communes à ses membres et qui les distinguent. En second lieu, l'appropriation d'un sport par une société donne lieu à de subtiles adaptations. Nous sommes là au cœur du concept d'ethnomotricité ${ }^{4}$, qui s'appuie directement sur les travaux de M. Mauss (1950 [1934]). En effet, tout comme une culture génère des techniques du corps qui lui sont propres, elle imprime à chacune des ses pratiques la marque de sa spécificité. Appliqué au sport, nous remarquerons par exemple que les joueurs de tennis issus des pays latins sont plus adeptes de la terre battue que ceux de culture britannique, plus friands du jeu sur gazon ${ }^{5}$. De la même manière, ne parle-t-on pas couramment de "l'école brésilienne » au football et de ses contrastes avec le «style allemand»? Enfin, ce spectre d'appropriation se trouvera démultiplié dès lors que l'on s'attachera à une échelle individuelle (style de jeu, répertoires tactique et technique, innovations...). Les pratiques ludomotrices seront donc envisagées ici comme de puissants vecteurs de socialisation, d'identification et de différenciation, l'action étant avant tout action sur soi.

Afin de mettre en ordre la diversité de pratiques ainsi énoncée, une analyse des aspects structurels et organisationnels permettra de dégager des différences de rapports à l'espace, au temps, à autrui et aux objets. Dans le sport par exemple, sur le plan interactionnel, seul prime le gain final et s'il y a coopération, celle-ci est au service de l'opposition : comme le souligne $\mathrm{B}$. Jeu, le sport « établit des règles où l'affrontement est érigé en valeur. On s'y rencontre même tout exprès pour s'opposer » (1992 : 22). D'autres activités ludomotrices en revanche laissent la

4. P. Parlebas définit l'ethnomotricité comme le " champ et [la] nature des pratiques envisagées sous l'angle de leur rapport à la culture et au milieu social au sein desquels elles se sont développées» (Parlebas 1999: 145).

5. Sur cet aspect, cf. Lesage 2006.

6. Jeux paradoxaux. 
part belle aux relations ambiguës et ambivalentes ${ }^{6}$; les relations sont instables et évoluent au gré des stratégies et des désirs des participants, comme lorsque l'adversaire peut devenir partenaire au cours de la partie (la balle assise, l'épervier...). Ceci nous amène à considérer que les pratiques physiques doivent s'analyser au regard de plusieurs logiques interdépendantes. La première concerne le fonctionnement et les mécanismes internes des pratiques (leur logique interne) qui sont liés à un système de contraintes déterminé par les règles du jeu. Cette logique exprime alors le rapport indissociable qu'entretient l'individu agissant avec l'environnement spatial, matériel ou humain ${ }^{7}$. Néanmoins, la logique interne à elle seule ne suffit pas à rendre compte de tous les tenants du fait social que nous étudions ici. Aussi, aux contraintes structurelles de la première, viennent se fondre deux autres logiques empreintes des normes et valeurs propres à la culture d'émergence de la pratique. Ainsi nous distinguons d'une part la logique organisationnelle (degré d'institutionnalisation, cadre compétitif ou non, enjeux économiques, médiatisation, spectacularisation, etc.) ; d'autre part, nous accordons une importance centrale à la logique individuelle du pratiquant, puisque celui-ci, pierre angulaire de ce système d'analyse, n'est pas une coquille vide au service des règles : il porte en permanence son propre bagage identitaire. Il dispose d'une part d'autonomie et n'est pas entièrement prédéterminé par les logiques des organisations instituées: il n'est pas que le produit du jeu et son pouvoir d'initiative reste prépondérant.

C'est au travers de ce filtre d'analyse, qui conjugue l'étude du pratiquant et des structures dans lesquelles il agit, que nous allons explorer l'identité jouée dans les jeux et les sports afin de mieux appréhender les conditions d'expression et d'émancipation du joueur agissant. Par conséquent notre contribution se fonde sur l'analyse des règles explicites des mécanismes internes des jeux et des sports, et des codes implicites du joueur.

Dans un premier temps, nous nous attacherons à explorer les traits distinctifs de la logique interne des pratiques physiques et des conséquences qu'ils entraînent sur l'accomplissement des conduites motrices et sur l'épanouissement et la construction de l'individu

7. La logique interne d'une pratique ne doit pas être confondue avec les règles du jeu; elle s'appuie sur ces dernières pour définir les rapports qu'entretient le pratiquant avec son espace de jeu, les impératifs temporels, autrui et les objets, tout cela faisant système. 
(personnalité, sociabilité, affirmation de soi, attitude citoyenne, etc.). Dans un second temps, nous étudierons la logique individuelle du joueur : il s'agira en somme de procéder à l'analyse de la sphère subjective dans un contexte objectivable et contraignant. Nous tenterons de montrer qu'il se dégage une intelligence singulière à ne pas négliger au cours de la pratique physique, l'intelligence motrice qui participe au développement de l'individu. Enfin, puisqu'il apparaît que le dispositif normatif imposé par les règlements sportifs ne sont pas simplement des carcans verrouillant le pratiquant mais bien un cadre lui offrant un univers de possibles, il semble pertinent de mettre en relief les aspects éminemment particuliers et différentiels des modes d'appropriation d'une pratique, tant sur le plan moteur que symbolique. Pour ce faire, nous circonscrirons l'analyse aux mécanismes implicites en jeu dans la construction identitaire du pratiquant.

\section{Les mécanismes internes des activités ludomotrices}

Les jeux et les sports se différencient essentiellement par leur degré d'institutionnalisation. En effet, ils restent des jeux tant qu'ils ne sont pas exercés sous la tutelle d'instances officielles à visée compétitive. Ce processus social de transformation qui augure le glissement de certains jeux vers le sport au cours du temps peut être caractérisé par la notion de sportification $»^{8}$ des pratiques physiques (d'autres auteurs emploient le terme de sportivisation), qui se manifeste dès lors que ces dernières sont prises en charge par une instance officielle et qui s'inscrivent dans le cadre de compétitions.

Par ses traits de logique interne, le sport et ses dirigeants domestiquent et standardisent le milieu physique et mondialisent la pratique physique afin qu'à n'importe quel endroit de la planète les records puissent être comparables et donc les pratiquants comparés. La conformité de l'espace, du temps, des instruments et des techniques demeure la marque de fabrique du sport.

En outre, un contrat ludique s'instaure entre le joueur et l'instance fédérale de sa discipline, qui fixe et gère son système de règles. L'acteur du jeu s'engage à respecter la règle qui lui est imposée, mais il jouit en

8. Par le processus de sportification, un jeu sportif aboutit à la mise en présence des quatre critères inhérents au statut de sport : règles, action motrice pertinente, prise en charge institutionnelle et compétition (Parlebas, 1981). 
même temps des nombreuses possibilités tactiques que lui offre ce système (Parlebas 1986) ${ }^{9}$. Dans une optique comparable, C. Duflo (1997 : 214) parle d'une « légaliberté », le jeu étant, pour ce philosophe, l'invention d'une liberté par et dans une légalité et limitée par une clôture temporelle qui "délimite la durée durant laquelle les règles valent et la légaliberté existe et s'exerce».

Les systèmes de contraintes et de possibilités qui dépendent des règles officielles du sport vont révéler l'expressivité de l'individu agissant. Le pratiquant se façonne ici dans une mise en jeu corporelle en se confrontant à lui-même, aux autres et au milieu physique.

Sur ce dernier critère, le sport privilégie, à l'instar de notre société moderne, l'accomplissement moteur dans un milieu physique plutôt stable, artificiel et standardisé. Dans ce contexte d'évolution, si le sportif agit seul dans son espace d'accomplissement normalisé et dénué d'incertitude, il vise l'excellence motrice et l'exploit magnifié en sollicitant plutôt une forte dépense énergétique - un 100 mètres d'athlétisme par exemple - ou une extraordinaire coordination motrice lors de la réalisation de figures gymniques.

Dans le sport de haut niveau (l'élite des pratiquants), le sportif met à rude épreuve son corps pour s'imposer, reflet de la réussite sur soi et sur les autres. Le culte de la performance (Ehrenberg 1991) peut augmenter la réussite de soi, développer l'identité personnelle et asseoir une place dans la société ${ }^{10}$. Ce façonnage corporel et psychologique, propre au haut niveau, pousse les sportifs dans leur quête d'ascétisme à défier les normes et les limites du possible. R. Redeker (2008) évoque pour sa part une véritable «déshumanisation » du sportif de haut niveau, ce qui n'est pas sans questionner le concept d'identité. Dans le cadre des règles d'une activité physique qui prescrit la pratique au sein d'un milieu plus naturel ou sauvage, le pratiquant est amené à affronter la nature, son imprévisibilité, voire sa dangerosité (course en descente en canoë-kayak ou en vol à voile). Néanmoins, ces pratiques sportives sont ici les moins nombreuses; de surcroît, dans certains sports comme le canoë-kayak, l'instance officielle choisit parfois de domestiquer le milieu naturel en créant des torrents artificiels (J.O. de Sydney, 2000).

9. Faisant référence au Contrat social de J-J Rousseau (1762), P. Parlebas énonce "un système de contraintes librement accepté " (1981: 101).

10. Notons que ces points positifs peuvent s'accompagner de revers. Alain Ehrenberg souligne par exemple la fragilité du sportif lorsque ses objectifs ne sont pas ou plus atteints. 
Les jeux de pleine nature sont davantage l'apanage des jeux de loisirs (traditionnels ou activités informelles) qui favorisent une culture hédoniste. On peut aussi évoluer en co-présence d'autres pratiquants (ski, surf, jogging en forêt, etc.) sans pour autant que les actions de chacun nécessitent d'en découdre ou d'accomplir une action instrumentale avec eux. La pratique en milieu naturel dénuée d'adversité et de compétition favorisera alors le lien social, le «vivre ensemble » et donc développera l'identité collective puisque l'affrontement se focalise sur la nature plutôt que sur l'homme. En l'occurrence, la cohésion socio-affective et la sociabilité seront d'autant plus fortes que le projet collectif se réalise dans un milieu physique incertain, hostile et sauvage, qui demande la coopération active de tous les acteurs pour atteindre l'objectif fixé (Parlebas 1986 ; Laporte 2005).

Les règles codifient également les interactions avec les autres pratiquants en favorisant une communication originale, non pas verbale comme à l'accoutumée, mais une communication motrice (Parlebas 1981). La logique interne induit un mode de relation à autrui (solidarité/ antagonisme) rendant manifeste une forme particulière de socialisation ; l'intériorisation de ces rapports au(x) partenaire(s) et a(ux) adversaire(s) s'opère principalement de façon tacite dans l'action générant un " inconscient collectif et institutionnalisé ». Le sport, eu égard au système de contraintes et au cadre compétitif, impose aux participants de maîtriser l'environnement physique et humain pour mieux le vaincre. Autrement dit, le pratiquant doit se dominer dans l'action pour, in fine, mieux dominer l'autre ou les autres, que ce soit sous forme de duel d'individus (sports de raquette ou de combat) ou d'équipes (sports collectifs). De fait, dans une société orientée vers la méritocratie, la pratique du sport peut être un bon vecteur de réussite en «apprenant à gagner »(Collard 2004). Le pratiquant se construit ainsi sur une expressivité individuelle et collective fondée sur la capacité à obtenir un gain, à être dominant et performant pour atteindre l'objectif fixé. Toutefois, tous les jeux ne sont pas strictement compétitifs dans toutes les sociétés (Lévi-Strauss 1962). La relation à l'autre privilégie alors la subtilité et la richesse des échanges corporels et des codes informels (Goffman 1974, 1991) : le jeu est alors le lieu d'affirmation des identités individuelles et collectives et la sphère socio-affective est alors pleinement sollicitée (jeux rituels, jeux paradoxaux). Force est de constater que la surreprésentation du sport nous renseigne sur un des 
ressorts culturels de nos sociétés occidentales quant à la prédilection accordée à ce type d'interactions.

De manière résumée, les conduites des joueurs sont soumises à l'influence directe des codes plus ou moins coercitives selon le type de situation jouée. Au travers de ces systèmes de contraintes, les acteurs vivent des situations ludomotrices singulières, distinctes des autres pratiques sociales. La mise en jeu corporelle au cours de ces pratiques physiques permet de prendre des risques, de connaittre ses limites, d'oser affronter autrui, de ruser, de tisser des liens sociaux; elle favorise aussi la recherche d'émotions particulières (l'«ilinx » selon Caillois 1958). De fait, elle contribue activement au développement de la personnalité (sur les plans émotionnel, affectif, cognitif, relationnel ou expressif), à la santé (physique, mentale et sociale), à la diversité culturelle de la société (où cohabitent diverses formes sociales de pratiques physiques). L'activité pratiquée se révèle alors être structurante bien au-delà du simple temps passé sur le terrain et modèle une certaine façon d'être au monde à laquelle le sujet est initié et qu'il incorpore dans le même mouvement.

Néanmoins, l'acteur n'est pas uniquement le jouet des règlements, ni un automate sur lequel les rouages et les mécanismes internes des sports imposent une manière de vivre et de s'exprimer. Il vit les situations, guidé selon ses traits de personnalité, ses émotions, ses représentations, son vécu, son marquage familial et social. Au cœur de la logique interne d'une pratique physique, le sujet engage donc sa logique individuelle originale, son intelligence motrice (Parlebas 1999). Celle-ci s'exprime dans le cadre des contraintes et des possibilités offertes par les règles. L'incorporation de ce cadre laisse la liberté au joueur de réinterpréter la situation dans laquelle il évolue en se dotant de règles et de codes d'action afin d'être efficace. Selon le type de situations, celui-ci utilise soit une intelligence motrice qui valorise le subtil décodage du milieu physique et humain, l'initiative et la décision motrice ou encore les relations socio-affectives (activités de pleine nature ou des jeux sportifs avec des adversaires) ; soit, dans les activités dénuées d'incertitude, une intelligence motrice qui valorise la sensibilité kinesthésique, la prise des repères proprioceptifs, la maîtrise affective. Ainsi, l'intelligence motrice place le joueur comme un véritable stratège. C'est pourquoi un simple regard sur les comportements observables du pratiquant - ses gestes et attitudes - ne suffit pas à rendre compte de ce qui se joue réellement. L'acteur «se met en jeu» : son identité, son 
intentionnalité sont au cœur des actions qu'il déploie. En revanche, l'analyse de ses conduites motrices nous renseigne sur l'action en tant qu'elle est porteuse de signification (pour lui-même et autrui) : élaboration et interprétation de codes et combinaisons, perceptions, émotions, sentiments, motivations, etc. Maître de son libre arbitre, il opère des choix rationnels qui peuvent le distancier du modèle archétypal dessiné par l'institution, ces choix pouvant même le conduire à faire fi des impératifs règlementaires. Souvenons-nous notamment de la polémique qui a soulevé le monde footballistique suite à la « la main de Dieu » de Diego Maradona ou plus récemment de celle de Thierry Henry ${ }^{11}$; ces deux exemples illustrant bien la prépondérance que peuvent prendre les logiques individuelles sur la logique du contrat règlementaire.

La compréhension du joueur ne peut donc se limiter à l'étude des règles explicites du jeu pratiqué ; il n'est pas enfermé dans un moule structurel annihilant sa liberté d'expression. Il existe une marge de liberté entre l'acteur et le système (Crozier et Friedberg 1977) dont les sportifs peuvent tirer profit, à l'instar de Dick Fosbury dont le saut dorsal rendu célèbre aux J.O de Mexico en 1968, révolutionna la discipline du saut en hauteur. Autrement dit, il existe du «jeu» dans les mécanismes internes des pratiques physiques; et celui-ci, bien observé, peut aider à différencier les pratiquants et à pénétrer leur sphère intime.

\section{Logique individuelle du joueur et construction identitaire}

Au-delà des règles explicites dont le rôle dans l'élaboration identitaire a pu être mis en évidence, la part originale de l'individu agissant nous amène à envisager les mécanismes implicites dans les pratiques ludomotrices. Afin de spécifier notre propos, nous focaliserons notre analyse au contexte sportif.

11. En effet ces deux champions de football ont violé le règlement à l'occasion respectivement, de la Coupe du Monde 1986 et du match de qualification pour la Coupe du Monde 2010. L'un comme l'autre ont suscité une action décisive dans l'obtention d'un but en interceptant le ballon de la main. Il est intéressant de noter, que ces actions marginales ont été réalisées alors même que leur statut de capitaine est censé leur conférer le rôle de garant des «valeurs du football».

12. Extraits d'une enquête de terrain réalisée par M. Level (thèse de doctorat en cours). 
Pratiquer assidûment une activité sportive c'est s'engager dans une logique particulière régissant "un monde à part », "un univers », "une famille $»^{12}$. L'imprégnation émanant des mécanismes implicites à l'œuvre à l'occasion d'un investissement prolongé dans un sport se situe à l'interface du collectif et de l'individuel. Le collectif d'abord, puisque les normes sont produites par le groupe de pairs, agents et acteurs d'une culture commune ; l'individuel ensuite, car chaque sujet « reprend à son compte » cet univers et le fait sien en même temps qu'il le transforme, modelant et exprimant par là son identité.

Ainsi, les pratiquants d'une même activité semblent-ils converger vers des valeurs, des normes et des représentations partagées. Cette appropriation de codes implicites trouve particulièrement sa réalisation au travers de la construction d'une image du corps façonnée par et pour la pratique. En effet, le travail, les maternités, la maladie, l'alimentation, comme le sport, marquent les corps, les plient, les transforment; et dans la chair se gravent les empreintes de l'histoire du sujet. Aussi, le travail sportif remodèle-t-il le corps anatomique comme le corps subjectif pour répondre aux exigences de performance. Lévolution du seuil de tolérance à la douleur ou la construction d'un idéal corporel sont deux illustrations particulièrement édifiantes du façonnage identitaire opéré de façon plus ou moins insidieuse par la pratique sportive sur ses adeptes. En effet, la recherche de performance oblige l'athlète à jongler au mieux avec son capital corporel - c'est-àdire maximiser l'usage du corps sans le «cramer», pour reprendre le jargon indigène - tout en évitant les blessures, ce qui l'amène le plus souvent aux limites du surentraînement. Par ailleurs, si nous ne pouvons douter que le sportif, peut-être mieux que quiconque, est à l'écoute de son corps ${ }^{13}$, la souffrance physique est admise d'emblée, consentie, voire recherchée par l'athlète qui se transcende dans l'effort. Ainsi, au-delà des contacts physiques parfois très " rugueux » prévus par les règlements, les douleurs sourdes, manifestations de l'usure du corps liée aux innombrables répétitions, sont le lot quotidien du pratiquant. Éduqué au «goût de l'effort », les courbatures sont pour lui le signe du travail fourni et présagent les résultats à venir. Il n'est d'ailleurs pas rare qu'un compétiteur taise sa douleur, préférant souffrir que de manquer une compétition majeure, allant jusqu'à dissimuler ses maux auprès de ses

13. Le sportif étant formé à écouter son corps, relayé pour cela par une équipe médicale très présente afin de s'assurer de l'efficacité et la longévité de son « outil de travail ». 
entraîneurs. Les marques visibles (bleus, cicatrices...) peuvent parfois même être les signes convoités de l'appartenance à une identité collective et revendiqués comme les preuves de l'abnégation, voire du sacrifice pour l'équipe ${ }^{14}$.

L'élaboration collective d'un certain idéal corporel qui définit la norme est un deuxième exemple des mécanismes implicites d'élaboration identitaire au cœur des pratiques sportives. Le grand, le petit, le mince, le gros, le féminin et le masculin se déterminent de façons différentes et s'intériorisent individuellement et de manière différenciée par le corps en mouvement au sein de chaque pratique. Le modèle archétypal prescrit les qualités définissant le «bon pratiquant », voire propose un morphotype ; aussi, les gymnastes de gymnastique rythmique par exemple, recherchent-elles une souplesse hors norme combinée à une musculature longiligne par opposition aux gymnastes aux agrès.

Les données empiriques (Level à paraitre) réalisé entre autres auprès de basketteurs, nous permet d'appréhender l'importance du sentiment éprouvé par les joueurs d'appartenir au «clan basket » au sein du champ sportif et le prima donné à cette facette de leur identité. Les sportifs interrogés témoignent à l'unanimité de l'enracinement profond de l'activité motrice dans la "culture basket », des diverses affiliations qui en découlent et de la distinction entre ceux qui «en sont " et les autres ${ }^{15}$. Les acteurs d'une même pratique forment ainsi une communauté autour de référentiels reconnus et partagés: des mythes (mythe des origines en surf par exemple ou héros populaires tels que Michael Jordan ou Zinedine Zidane), des goûts (musicaux, vestimentaires...), des lieux («spots» pour le roller, magasin «Made in sport » au basket ou quartier de l'Odéon à Paris, véritable fief pour les sorties nocturnes des rugbymen parisiens).

Ce phénomène est notamment accentué par ce que E. Goffman caractérise d' "All-time-in », souvent corrélé à une forme de retrait par rapport à l'environnement social extérieur à la pratique. Celle-ci se distancie alors de la simple activité pour devenir un art de vivre. Pour

14. L'ouvrage de Y. Le Hénaff et S. Héas (2007), Tatouages et cicatrices, décors sportifs, fournit de nombreux exemples en rugby et free ride.

15. Notons à ce propos que l'opposition à l'Autre, c'est-à-dire dans ce cas celui qui n'appartient pas à la sphère du basket, est autant facteur d'identité que l'identification aux pairs. 
certains, venir à l'entraînement c'est tout à la fois venir travailler (pour soi-même et très souvent en plus pour le club en entraînant une autre équipe), rejoindre ses amis et parfois même son conjoint.

Enfin, l'appartenance au groupe et la reconnaissance des pairs s'identifie grâce au recours à des signes distinctifs plus ou moins ostensibles, véritables emblèmes de subjectivation, dont un des supports majeurs est la culture matérielle ${ }^{16}$. Ainsi, aux objets inhérents à l'action motrice, dont l'usage est réglementé par la logique interne, se combinent une multitude d'objets connexes qui gravitent autours de la pratique sportive et la prolongent au quotidien, participant à la sociabilité du sujet en tant que sportif. La présence de mini-ballons dans la chambre, en porte-clé ou accroché au rétroviseur, d'affiches, de vidéos mettant en scène les exploits des champions et qui circulent entre les pratiquants, de T-shirts à l'effigie du club ou d'une compétition réalisée, ou encore une mode vestimentaire (streetwear «baggy» ou la marque Eden Park...) en sont des exemples récurrents. Ainsi, A. Mottiaux (2005) démontre-t-elle que la garde-robe des coureurs est faite de T-shirts mentionnant le club d'appartenance et les courses auxquelles ils ont participé en vue d'afficher la participation, l'engouement et l'appartenance à une communauté de destin liée par la communion à travers l'expérience sportive.

Tous ces objets participent de la culture matérielle incorporée du sujet et nous montrent à quel point son identité, bien que plurielle, apparaît comme éminemment rivée à son sport. Dans l'action, la question des usages de l'instrument sportif nous amène à considérer que ce dernier ne prend sens que s'il est combiné à l'action motrice du sportif agissant dans un cadre définitoire. Autrement dit, action motrice et engins sont étroitement imbriqués, ce qui signifie que l'étude de la motricité ne peut faire fi de l'analyse du processus d'incorporation des objets par le pratiquant (cf. Warnier 1999, 2009). Or, cette appropriation différenciée fait partie intégrante des mécanismes implicites de production du sujet sportif et d'appropriation de son univers.

16. J.-P. Warnier définit la culture matérielle comme «tous les objets matériels, quels qu'ils soient, manufacturés ou non, vivants ou non, dont la statique et la dynamique sont plus ou moins incorporées dans les conduites motrices. A cela s'ajoute tout l'environnement qui sert de cadre à ces actions » (1999: 26). 
En effet, par ce processus, principalement de l'ordre de la mémoire implicite et des mécanismes infra-conscients, le sujet se singularise, façonne une identité, tout autant qu'il s'inscrit dans son groupe de référence. Il opère une multitude de choix et de stratégies (choix du matériel, bricolages et personnalisations en tous genres ${ }^{17}$ ), exprimant et développant ainsi sa personnalité et son individualité. De la même manière, les conduites motrices créées, nourries d'un répertoire moteur et d'une sensorialité éminemment personnels sont facteurs d'identité, au même titre qu'un trait de caractère ou un attribut physique.

Sur ce dernier point, il convient de remarquer que si le concept d'incorporation renvoie le plus souvent à une intériorisation des conduites motrices imbriquées aux artefacts qui les sous-tendent et des manières d'être et d'agir qui passeraient directement dans les corps sur le mode de la communication silencieuse, de l' "apprendre par corps » (Faure 2000), il serait erroné d'exclure la médiation langagière de ce processus. De fait, si les consignes, les métaphores explicatives et les corrections ponctuent toute séance d'entraînement, chaque pratique sportive possède un code langagier qui peut complètement exclure le « profane». Les sportifs doivent donc assimiler une véritable grammaire propre à leur pratique dont l'acquisition fait partie intégrante du processus de socialisation et d'individuation du sujet. Le partage d'un vocabulaire participe alors du phénomène d'inclusion et d'exclusion qui dessine les contours d'une communauté.

Dans le rapport entre le joueur et l'instrument qu'il manipule, une symbolique basée sur le vécu ludosportif (et a fortiori compétitif) s'ajoute à l'aspect proprement technique de l'expérience du maniement. Ainsi,

17. Les exemples de bricolages et de décoration du matériel sont pléthores (dessins, peinture, autocollants, grip, mousse...). Ce phénomène d'appropriation de l'objet par le sportif, en procédant à une série de modifications, est tel que certains équipementiers ont décidé de fournir des engins vierges (par exemple des rubans ou des cerceaux blancs en gymnastique rythmique) afin de laisser libre cours au désir des pratiquants. Décorer, bricoler, aménager : l'objet est ainsi singulier, investi d'affects et plus efficient, autant de personnalisations hautement matrice de subjectivité.

18. Le couteau norvégien en est un autre exemple. En effet, selon Bjarne Rogan (1996), cet outil a le pouvoir de rassembler par un mouvement d'identification nationale à un passé lointain, mythique et héroïque, par la nostalgie de la société préindustrielle. Outil, arme et parure virile du paysan libre et indépendant, "l'homme sans couteau est un homme sans vie ». Le couteau apparaît comme un morceau de Norvège, véritable symbole de la conscience identitaire et objet de sociabilité (les clubs d'amateurs et collectionneurs). 
les objets pourraient échapper à leur matérialité pour endosser une charge affective, porter une signification symbolique ou se teinter d'humanité. Les objets constituent alors de formidables supports à la mémoire collective ${ }^{18}$ d'une part (souvenir d'une compétition gagnée ensemble, d'un stage, d'un entraîneur, d'un champion mythique...) et à la mémoire du sportif d'autre part ${ }^{19}$. En effet, l'engin semble contenir une histoire, celle de son propriétaire. Telle la madeleine de Proust, le ballon par exemple, porte en lui «l'édifice immense du souvenir »: il peut tout aussi bien suggérer les débuts dans la pratique et la genèse d'une passion, les motivations qui poussent à persévérer, que l'entraîneur qui a su motiver ou encore une grande réussite. De surcroît, les instruments sont très souvent gardés précieusement par le sportif, même quand ils ne sont plus en usage tant ils sont associés aux temps forts de sa vie et jalonnent sa construction : traces de sa carrière, ils sont de véritables repères identitaires. Ainsi le joueur de tennis Arnaud Boetsch confiait-il dans une interview au journal L'Equipe ${ }^{20}$ qu'il conserverait chez lui son ancienne raquette "parce qu'elle représente d'innombrables souvenirs $»^{21}$.

Certaines conduites rituelles peuvent alors apparaître, comme le fait de ne pas vouloir jouer avec un autre instrument ${ }^{22}$ que celui avec lequel s'est instauré un vécu particulier (eu égard à des victoires probantes). Nous pouvons parler dans ce cas d'un rapport de type fétichiste, l'instrument se voyant attribuer une valeur positive (celle d'un objet porte-bonheur) par son propriétaire. L'histoire personnelle façonnée entre le joueur et l'objet renvoie manifestement au concept

19. Le lecteur pourra consulter à ce propos l'ouvrage dirigé par Octave Debary et de Laurier Turgeon (2007) qui regroupe les contributions de nombreux auteurs qui abordent la relation avec les objets sous l'angle de l'identité, de la mémoire ou de la construction des rapports sociaux.

20. Propos recueillis par Hervé Bombrun, le 25 Avril 1995.

21. Nous pouvons à ce propos noter que la valeur symbolique conférée à l'objet sportif prend fréquemment la forme d'une personnification renvoyant dans des cas extrêmes au concept d'animisme. Ainsi, si certaines gymnastes vont jusqu'à donner des noms à leurs engins, nombreux sont les sportifs à prêter une intentionnalité à l'objet, comme si celui-ci pouvait choisir d'être un allié ou au contraire gêner la performance.

22. Maillot au numéro fétiche, slip porte-bonheur, paire de chaussures, bandeau, raquette, pagaie...Le joueur de tennis Yannick Noah par exemple, a-t-il confié porter lors de sa finale de Roland Garros en 1983 sa «tenue fétiche » avec laquelle il avait auparavant remporté de nombreuses victoires. 
d' « objectification » initié par D. Miller (1987) et dont P. LaburtheTolra et J.-P. Warnier soulignent l'importance dans le rapport sujet-objet : un «processus total par lequel le sujet et l'objet se construisent ensemble et l'un pour l'autre» $(1993: 347)^{24}$.

Par ailleurs, la mise en place systématique de routines individuelles sans cesse reproduites et composées d'une série de gestes organisés en un temps et un espace donné de façon plus ou moins cérémonielle (dans les vestiaires, avant d'entrer sur le praticable, avant un lancer franc...) favorise aussi bien la concentration et la mémorisation, et par conséquent l'efficacité, que la production du sujet par la création de conduites motrices qui lui sont propres. Enfin, les habitudes collectives, telles que le fait de se taper dans les mains ou sur les fesses, ou encore l'ordre de passage à l'échauffement d'un match, ont entre autres fonctions de constituer le clan et de le souder face à la bataille à mener. L'intégration d'un nouveau joueur au groupe passe d'ailleurs par son appropriation des rituels collectifs. Ainsi, par l'acquisition de comportements reconnus par le groupe, le sujet renforce-t-il son sentiment d'appartenance.

En dernière analyse, la pratique d'un sport suppose un double processus d'intégration de codes de comportements et d'une certaine morale explicitement codifiés par les règlements, et d'incorporation d'une culture particulière fondée sur l'élaboration et le partage de normes, de représentations et de conduites qui sont assimilées collectivement et individuellement de façon plus tacite. Mais au-delà, cet univers offre un espace de liberté, de créativité et donne lieu à une multitude d'innovations singulières. Si les techniques du corps sont transmises par l'éducation et l'imitation, une fois celles-ci incorporées, le sujet agissant se singularise en opérant par exemple une série de choix. De plus, l'originalité des morphologies et des expériences passées et présentes sont autant de paramètres qui entrent en compte dans l'appropriation des techniques. Enfin, mais ceci découle des deux premières idées, l'ensemble des bricolages réalisés par les sportifs euxmêmes pour personnaliser leur engin dans le but de le rendre plus adapté à une façon de faire tout à fait propre, offre un espace d'innovation et de subjectivité. Les techniques s'incorporent donc à travers le prisme des biographies et des morphologies individuelles (qui se font très souvent écho). Aussi une ancienne blessure au poignet influencera-telle la façon de manipuler l'instrument. Si certains aspects singularisant une gestuelle peuvent être considérés comme des défauts et 
« rééduqués », nous pourrions objecter que les sportifs évoluant au plus haut niveau sont également porteurs de différences individuelles. Il paraît donc vain de vouloir uniformiser une technique qui serait la meilleure pour tous, tandis que la prise en compte des particularités individuelles pourrait en faire des qualités exploitables. Ainsi, les individus expriment-ils très clairement leur singularité à travers leur «coup de pagaie» ou leur «style de jeu».

Ensuite, la perception qu'un individu a de lui-même, et de son corps en particulier, participe étroitement de la construction de son identité. Or, la pratique d'une activité physique apparaît comme un bon moyen de se construire corporellement (morphologie et conduites motrices) et dans le même temps de développer une meilleure connaissance de soi, dans ses possibilités sensorielles et d'action, notamment sous l'angle des compétences. Les potentiels d'expressivité par le corps sont ainsi favorisés, ce qui permet en outre d'être pleinement conscient des contours de son être. De surcroît, pour certaines pratiques s'ajoute la particularité d'une visée artistique, donc d'expression, et malgré les contraintes réglementaires fortes, le praticable ou la patinoire apparaissent comme des espaces de "liberté » et d'affirmation de soi.

D'autre part, le sport est un monde à part régi par des règles qui ne sont pas les mêmes que celles du reste du champ social. Aussi, comme nous l'avons évoqué en première partie, ces pratiques offrent-elles la possibilité à l'individu d'exprimer une autre facette de ce qu'il est. Par exemple, la violence ou l'agressivité font actes de déviance dans notre société tandis qu'elles sont admises, voire requises (réellement ou symboliquement) dans le domaine sportif. Ne parle-t-on pas d'ailleurs du vestiaire comme d'un lieu de «métamorphose » de l'état d'individu à celui de sportif?

Enfin, la persévérance dans l'activité, malgré les efforts et les concessions que cela suppose, n'est pas étrangère au plaisir narcissique de trouver un domaine de reconnaissance lié aux bonnes performances ; car au-delà de la recherche de compétence et de légitimité, le plaisir éprouvé à jouer, et dans les limites de chacun, à bien jouer, reste la raison première de la pratique et un trait fondamental de l'identité du sportif.

\section{Conclusion}


Le sport se pose comme un système de contraintes qui présente de multiples interstices de liberté relatifs au degré d'institutionnalisation de la pratique. L'évolution des normes et des valeurs de la société d'accueil au fil du temps et les caractéristiques individuelles amèneront les pratiquants à osciller entre des pratiques plus compétitives et des activités à visée hygiéniste ou hédoniste. Plus une activité est avancée dans son processus de sportification, plus le sportif est astreint à un grand nombre d'exigences réglementaires. Cette contrainte librement consentie relève d'un contrat explicite passé entre l'instance officielle et le joueur. Ce pacte est primordial pour que s'établissent les relations entre l'acteur du jeu et les différents éléments du système, c'est-à-dire les traits de logique interne : rapports à autrui, à l'espace, au temps, aux objets ou encore au système de score.

Ainsi, selon le type de relations que le sportif noue avec son environnement spatio-temporel matériel et humain, sera par exemple favorisée la recherche de domination plutôt que la création de lien social (opposition/coopération) ou l'intelligence motrice plutôt que la quête d'excellence individuelle. Les traits de logique interne entraînent donc des conséquences sur l'accomplissement des conduites motrices et sur l'épanouissement et la construction de l'individu (personnalité, sociabilité, affirmation de soi, attitude citoyenne, etc.).

Dans ce contexte, les acteurs se nourrissent d'expériences ludomotrices singulières qui les plongent dans un cadre distinct des autres formes de pratiques sociales. Ainsi, l'agressivité et la douleur sont-elles valorisées lorsqu'elles sont bornées à l'aire de jeu tandis qu'elles sont proscrites en dehors. Le sport s'inscrit clairement comme un espace de libertés, d'innovations et de compétence, même si des notions relatives aux idées de souffrance, de défaite ou de domination ne peuvent être complètement éludées. De facto, ce vécu sportif est producteur de subjectivité.

Chaque société sécrète une formation corporelle en adéquation avec ses principes normatifs et axiologiques. Cette élaboration s'articule autour de mécanismes aussi bien explicites qu'implicites qui portent le sceau du collectif autant qu'ils sont nécessairement réinvestis de façon individuelle. Elle est donc productrice de structures communes tout autant que de différences singularisantes. Celles-ci font également l'objet d'une incorporation principalement tacite: par l'assimilation de codes de comportements et d'interactions avec le groupe de pairs ou de normes 
de représentations, la pratique sportive apparaît comme facteur de socialisation et creuset des identités. Jouer, c'est se produire soi-même comme joueur, à l'instar de la production de subjectivité à l'œuvre dans l'engagement politique ou religieux. En ce sens, s'adonner à une pratique ludomotrice engendre ce qui n'est pas sans rappeler les techniques de soi, proposées par M. Foucault (1984).

Pour conclure, plutôt que de considérer que ce que le sport gagne à être extrêmement codifié il le perd systématiquement au regard de la richesse induite par la diversité, il nous paraît plus pertinent d'appréhender le cadre normatif comme une partition que le sujet est libre de réinterpréter, donnant la part belle à sa subjectivité. 


\section{Bibliographie}

Bordes, Pascal, Luc Collard et Éric Dugas, 2007, Vers une science des activités physiques et sportives : la science de l'action motrice. Paris, Vuibert.

Brohm, Jean-Marie, 2006, La tyrannie sportive. Théorie critique d'un opium du peuple. Paris, Beauchesne.

Caillois, Roger, 1958, Les jeux et les hommes. Paris, Gallimard.

Callède Jean-Paul, 2007, La sociologie française et la pratique sportive (1875-2005). Pessac, Maison des Sciences de l'Homme et d'Aquitaine.

Collard, Luc, 2004, Sport et agressivité. Méolans-Revel, DésIris.

Crozier Michel et Erhard Friedberg, 1977, L'acteur et le système. Paris, Seuil.

Duflo, Colas, 1997, Jouer et philosopher. Paris, PUF.

Dugas, Éric, 2008, «Sport et effets éducatifs à l'école : de la violence à l'agressivité motrice ». International Journal on Violence and School, $5: 67-83$.

Elias, Norbert et Eric Dunning, 1994, Sport et civilisation ; la violence maitrisée. Paris, Fayard.

Ehrenberg, Alain, 1991, Le Culte de la performance. Paris, CalmannLévy.

Faure, Sylvia, 2000, Apprendre par corps. Socio-anthropologie des techniques de danse. La Dispute, Paris.

Foucault, Michel, 1984, L'usage des plaisirs. Histoire de la sexualité, II. Paris, Gallimard.

Goffman, Erving 1974, Les rites d'interaction. Paris, Minuit.

Goffman, Erving, 1991, Les cadres de l'expérience. Paris, Minuit.

Guillemard, Gérard, Jean-Claude Marchal, Martine Parent, Pierre Parlebas et André Schmitt, 1984, Aux 4 coins des jeux. Paris, Scarabée.

Jeu, Bernard, 1987, Analyse du sport. Paris, PUF.

Julien, Marie-Pierre et Céline Rosselin, 2005, La culture matérielle. Paris, La Découverte.

Laporte, Renaud, 2005, "Pratiques sportives et sociabilité ». Mathematics and Social Sciences, $170: 57-77$.

Laburthe-Tolra, Philippe et Jean-Pierre Warnier, 1993, Ethnologie, anthropologie. Paris, PUF.

Lesage, Thierry, 2006, Les jeux de paume et de raquette : filiations, logique interne et déterminants culturels. Thèse de Doctorat en sciences sociales. Université Paris Descartes. 
Level, Marie (à paraître), Etude comparée du processus d'incorporation de la dynamique des objets en kayak, gymnastique rythmique et basketball. Thèse de Doctorat STAPS, Université Paris Descartes.

Lévi-Strauss, Claude, 1962, La pensée sauvage. Paris, Plon.

Marchal, Jean-Claude, 1990, Jeux traditionnels et jeux sportifs; bases symboliques et traitement didactique. Paris, Vigot.

Mauss, Marcel, 1950 [1934], «Les techniques du corps ». Dans Sociologie et anthropologie. Paris, PUF : 365-386.

Miller, Daniel, 1987, Material culture and mass consumption. Oxford, Basil Blackwell.

Mottiaux, Aude, 2005, « Nudité et vêtements : évolution des symboles propres aux coureurs/euses à pied ». Dans Anne Roger et Thierry Terret (dir.), Sport et Genre, Tome 4. Paris, L'Harmattan : 49-64.

Parlebas, Pierre, 1981, Contribution à un lexique commenté en science de l'action motrice. INSEP, Paris.

Parlebas, Pierre, 1986, Éléments de sociologie du sport. Paris, PUF.

Parlebas, Pierre, 1999, Jeux, sports et sociétés : lexique de praxéologie motrice. Paris, INSEP.

Redeker, Robert, 2008, Le sport est-il surhumain? Paris, Panama.

Rousseau, Jean-Jacques 1972 [1762], Du contrat social. Paris, Hachette.

Warnier Jean-Pierre, 1999, Construire la culture matérielle: l'homme qui pensait avec ses doigts. Paris, PUF.

Warnier, Jean-Pierre, 2009, "Technology as Efficacious Action on Objects... and Subjects ». Journal of Material Culture, 14 (4) : 459 470. 